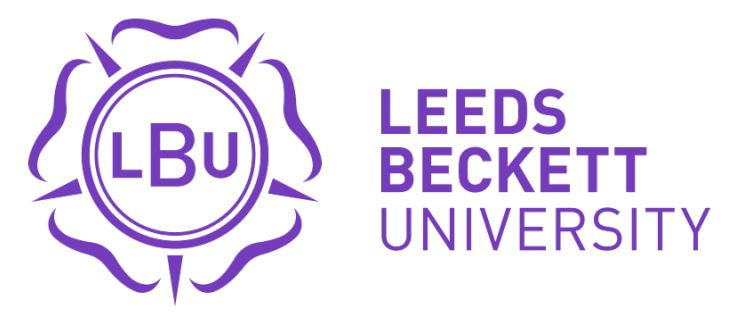

Citation:

$\mathrm{Li}, \mathrm{L}$ and Liao, F and Deng, J (2017) H Preview Control of a Class of Uncertain Discrete-Time Systems. Asian Journal of Control. ISSN 1022-2812 DOI: https://doi.org/10.1002/asjc.1466

Link to Leeds Beckett Repository record:

https://eprints.leedsbeckett.ac.uk/id/eprint/3341/

Document Version:

Article (Accepted Version)

The aim of the Leeds Beckett Repository is to provide open access to our research, as required by funder policies and permitted by publishers and copyright law.

The Leeds Beckett repository holds a wide range of publications, each of which has been checked for copyright and the relevant embargo period has been applied by the Research Services team.

We operate on a standard take-down policy. If you are the author or publisher of an output and you would like it removed from the repository, please contact us and we will investigate on a case-by-case basis.

Each thesis in the repository has been cleared where necessary by the author for third party copyright. If you would like a thesis to be removed from the repository or believe there is an issue with copyright, please contact us on openaccess@leedsbeckett.ac.uk and we will investigate on a case-by-case basis. 


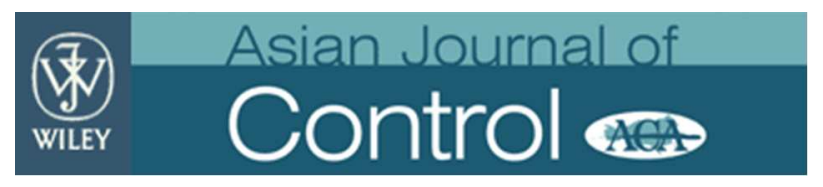

\section{Ho Preview Control of a Class of Uncertain Discrete-Time Systems}

\begin{tabular}{|c|c|}
\hline Journal: & Asian Journal of Control \\
\hline Manuscript ID & RR-16-0296.R2 \\
\hline Wiley - Manuscript type: & Regular issue: Regular paper \\
\hline Date Submitted by the Author: & 04-Oct-2016 \\
\hline Complete List of Authors: & $\begin{array}{l}\mathrm{Li}, \mathrm{Li} \text {; University of Science and Technology Beijing, School of Mathematics } \\
\text { and Physics } \\
\text { Liao, Fucheng; University of Science and Technology Beijing, School of } \\
\text { Mathematics and Physics } \\
\text { Deng, Jia; Leeds Beckett University, Leeds Sustanibility Institute }\end{array}$ \\
\hline Keywords: & $\begin{array}{l}\text { augmented error system, preview control, robust tracking, uncertain } \\
\text { system, observer, LMI }\end{array}$ \\
\hline Abstract: & $\begin{array}{l}\text { This paper investigates the problem of Ho preview tracking control with } \\
\text { robust performance for uncertain discrete-time systems. In order to avoid } \\
\text { applying the difference operator to the time-varying matrix, by taking } \\
\text { advantage of the difference between the system state variables, input } \\
\text { variables, and the corresponding auxiliary variables, instead of the usual } \\
\text { difference between system states, an augmented error system including } \\
\text { previewed information is constructed, which converts the tracking problem } \\
\text { into a regulator problem. A sufficient condition based on the free-weighting } \\
\text { matrices technique and the Lyapunov stability theory is derived for the } \\
\text { robust asymptotic stability of uncertain systems. Moreover, a state } \\
\text { feedback control law with preview action design method is obtained via } \\
\text { linear matrix inequality (LMI) approach. Based on these, a state observer } \\
\text { for preview control systems is formulated. Previewable reference signals } \\
\text { are fully utilized through reformulation of the output equation while } \\
\text { designing the state observer. The proposed construction method of } \\
\text { augmented error system is applicable to uncertain discrete-time system in } \\
\text { which the uncertainties are general. Also an integrator is introduced to } \\
\text { ensure the closed-loop system tracking performance with no static error. } \\
\text { The numerical results also show the effectiveness of the preview control } \\
\text { law for uncertain systems in the paper. }\end{array}$ \\
\hline
\end{tabular}




\title{
$H_{\infty}$ Preview Control of a Class of Uncertain Discrete-Time Systems
}

\author{
Li Li Fucheng Liao \\ School of Mathematics and Physics, University of Science and Technology Beijing, \\ Beijing 100083, China \\ Jiamei Deng \\ Leeds Sustanibility Institute, Leeds Beckett University, Leeds, UK LS2 9EN
}

\begin{abstract}
This paper investigates the problem of $H_{\infty}$ preview tracking control with robust performance for uncertain discrete-time systems. In order to avoid applying the difference operator to the time-varying matrix, by taking advantage of the difference between the system state variables, input variables, and the corresponding auxiliary variables, instead of the usual difference between system states, an augmented error system including previewed information is constructed, which converts the tracking problem into a regulator problem. A sufficient condition based on the free-weighting matrices technique and the Lyapunov stability theory is derived for the robust asymptotic stability of uncertain systems. Moreover, a state feedback control law with preview action design method is obtained via linear matrix inequality (LMI) approach. Based on these, a state observer for preview control systems is formulated. Previewable reference signals are fully utilized through reformulation of the output equation while designing the state observer. The proposed construction method of augmented error system is applicable to uncertain discrete-time system in which the uncertainties are general. Also an integrator is introduced to ensure the closed-loop system tracking performance with no static error. The numerical results also show the effectiveness of the preview control law for uncertain systems in the paper.
\end{abstract}

Keywords: augmented error system; preview control; robust tracking; uncertain system; observer; LMI

\section{Introduction}


The research question of preview control theory is formulated as follows: when the reference signal or exogenous disturbance is known or can be previewable, how can we take advantage of the previewed future desired output signal or disturbance signal to achieve the best control performance of a closed-loop system? Sheridan proposed the concept of preview control via three models [1]. Subsequently, for discrete-time constant coefficient linear systems, Katayama et al. constructed an augmented system that is equivalent to the original system [2], applying the difference in some degree between the state variables, tracking error variables. And based on optimal control theory, the optimal preview controller for the original system was obtained. Tomizuka [3] applied the same approach as that of Katayama et al. to continuous-time systems and derived an augmented error system similar to that of the discrete-time system. Finally an optimal preview controller was obtained by the extremum principle. Due to decades of research, preview control not only has made a major theoretical breakthrough (e.g., preview control theory of multirate systems, time-varying systems, continuous-time stochastic systems, and so on [4-7]), but it also has been used successfully in many other areas [8-10].

Up to now, most existing results for preview control theory are based on deterministic systems. However, in fact, uncertainty and external disturbance have become the essential parts of the control systems. Therefore, it is necessary to consider the robust stability and performance of the preview systems. Using $H_{\infty}$ and $H_{2}$ optimal control theories, various studies [11-14] have considered the control problems of preview control systems with external disturbances and given us the preview control design method. The game-theoretic approach was introduced into the continuous-time systems to propose the preview control problems and the state feedback and output feedback were both studied [15]. Subsequently, Cohen and Shaked [16] employed the method in [14] to discuss the robust preview control problem for discrete-time systems. Cohen and Shaked [17] proposed the robust $H_{\infty}$ preview control problem for norm-bounded uncertain systems based on the results of [15, 16]. Researchers in [18-20] applied the error system method in [21] to polytopic uncertain systems to consider the preview control laws design method problems.

Combining the discrete lifting technique with an auxiliary approach, an error system is constructed and the previewable reference signal is added to the state vector of the augmented error system. Consequently, the tracking control problem is transformed into 
the robust $H_{\infty}$ control problem. Based on this approach, the robust preview tracking control problem will be discussed. Two appropriate auxiliary variables are introduced to construct the error system. Therefore, this paper can avoid applying the difference operator to the time-varying matrix while deducting the formal system. Moreover, the problem over a finite-time interval that has been only discussed in $[6,12]$ can then be spread to the infinite time interval case. For the augmented error system, the state feedback controller and the reduced-order observer are derived, respectively. The gain matrix of the controller with preview action design can be transformed to solving linear matrix inequalities (LMIs). The effectiveness of the results is shown by numerical simulations.

Notations. $R^{n}$ denotes the n-dimensional Euclidean space and $R^{n \times m}$ is $n \times m$ matrix space, respectively. $A>0$ means that $A$ is positive definite. $A>B$ denotes $A-B>0 . A^{T}$ denotes the matrix transposition of $A . I$ denotes the identity matrix, and the number of rows and columns can be known from the context of the narrative. The symbol * stands for the transposed elements in the symmetric matrix, that is, $\left[\begin{array}{ll}X & Y \\ * & Z\end{array}\right]=\left[\begin{array}{cc}X & Y \\ Y^{T} & Z\end{array}\right] . \operatorname{sym}(A)$ denotes $A+A^{T}$.

In the following, we give the two lemmas that will be used.

Lemma 1 (Schur Complement Lemma [22])

Suppose matrix $\Omega=\left[\begin{array}{ll}\Omega_{11} & \Omega_{12} \\ \Omega_{12}{ }^{T} & \Omega_{22}\end{array}\right]$, where $\Omega_{11}$ and $\Omega_{22}$ are symmetric matrices and invertible, then the three conditions are equivalent as follows:

(i) $\Omega<0$;

(ii) $\Omega_{11}<0, \Omega_{22}-\Omega_{12}^{T} \Omega_{11}^{-1} \Omega_{12}<0$;

(iii) $\Omega_{22}<0, \Omega_{11}-\Omega_{12} \Omega_{22}^{-1} \Omega_{12}^{T}<0$.

Lemma 2 ([23]) For matrices $E$ and $G$ with appropriate dimensions, uncertain matrices $\Xi_{1}, \Xi_{2}, \cdots, \Xi_{s}$ that satisfy $\Xi_{i}{ }^{T} \Xi_{i} \leq I, \quad i=1,2, \cdots, s$ and arbitrary positive scalars $\varepsilon_{1}, \varepsilon_{2}, \cdots, \varepsilon_{s}$, the following LMI holds:

$$
E \Xi G+G^{T} \Xi^{T} E^{T} \leq E \Lambda E^{T}+G^{T} \Lambda^{-1} G,
$$

where $\Xi=\operatorname{diag}\left(\Xi_{1}, \Xi_{2}, \cdots, \Xi_{s}\right), \Lambda=\operatorname{diag}\left(\varepsilon_{1} I, \varepsilon_{2} I, \cdots, \varepsilon_{s} I\right)$. 


\section{Problem formulation and basic assumptions}

Consider the following uncertain discrete-time system:

$$
\left\{\begin{aligned}
x(k+1) & =[A+\Delta A] x(k)+[B+\Delta B] u(k)+[D+\Delta D] w(k), \\
y(k) & =C x(k),
\end{aligned}\right.
$$

where $x(k) \in R^{n}$ is the state vector, $u(k) \in R^{q}$ is the input control vector, $y(k) \in R^{q}$ is the output vector, $w(k) \in R^{l}$ is the disturbance vector, and $w(k) \in l_{2}, A, B, C$ and $D$ are constant matrices with appropriate dimensions. $\Delta A=\Delta A(k, x, \delta)$, $\Delta B=\Delta B(k, x, \delta)$ and $\Delta D=\Delta D(k, x, \delta)$ are uncertain matrices which depend on the time variable $k$, the state vector $x$, or some parameter vector $\delta$. In order to ensure the existence of the state observer of system (1), we assume that $(C, A)$ is observable. Other assumptions are as follows:

$$
\text { Assumption 1: }\left[\begin{array}{cc}
A-I & B \\
C & 0
\end{array}\right] \text { is invertible. }
$$

Assumption 1 is the standard assumption for the servomechanism design problem and shows that the nominal system $(A, B, C)$ has no zeros at $\lambda=1$.

The following is the commonly used assumption in preview control theory about the predictability of the reference signal:

Assumption 2: The $M_{R}$ future values, $r(k+1), r(k+2), \cdots, r\left(k+M_{R}\right)$ as well as the present and past values of the reference signal are available at each time $k$. The future values of the reference signal beyond the $k+M_{R}$ are zero, namely

$$
r(k+j)=0, j=M_{R}+1, M_{R}+2, M_{R}+3, \cdots
$$

where $M_{R}$ is the preview length of the reference signal.

And

$$
\lim _{k \rightarrow \infty} r(k)=r
$$

where $r$ is a known constant-vector.

It should be point out that (2) is the assumption for the reference signal which exceeds the preview length and (3) implies that the reference signal is an arbitrary time-varying function, except that it reaches a steady state. 
Remark 1: Theoretical research and practical examples have shown that the previewable signal has great influence on control effect of the closed-loop system only for a certain time period during which it exceeds the preview length impact is small; therefore, the reference signal is assumed to be a constant when it exceeds the preview length. In fact, a regular feedback control system does not consider the known future information of the previewable signal, or equivalently, $M_{R}=0$.

Assumption 3: There exist real constant matrices with appropriate dimensions $E_{i}, H_{i}, \quad(i=1,2,3)$ and uncertain matrices $\sum_{i}=\sum_{i}(k, x, \delta)$ such that

$$
\begin{gathered}
\Delta A=E_{1} \sum_{1} H_{1}, \Delta B=E_{2} \sum_{2} H_{2}, \Delta D=E_{3} \sum_{3} H_{3}, \\
\sum_{i}^{T} \sum_{i} \leq I .
\end{gathered}
$$

Remark 2: In Assumption 3, (4) shows that the uncertain matrices of system (1) satisfy matching conditions; (5) shows that uncertain matrices are norm bounded. It is easily seen from expressions $\Delta A, \Delta B$ and $\Delta D$ that the uncertainties are associated with the state vector or some unknown parameter and may be time varying in nature. Therefore, the uncertainties referred to in this paper are very general.

It should be noted that the assumption about the uncertain terms in [24] is adopted, rather than the commonly used assumption in [25]. As mentioned in Remark 2, the above assumption is more general. In designing the preview controller, if the uncertain matrices depend on $\delta$, then the usual difference method can be used to derive the augmented error system, see references [18-20]. On the other hand, if they are related to $k$, the method in [5] is applicable.

The nominal system of system (1) is

$$
\left\{\begin{aligned}
x(k+1) & =A x(k)+B u(k), \\
y(k) & =C x(k) .
\end{aligned}\right.
$$

Motivated by [26-28], we use system (6) to construct the following variables

$$
\tilde{x}(k)=x(k)-\bar{x}(k), \tilde{u}(k)=u(k)-\bar{u}(k),
$$

where $\bar{x}(k) 、 \bar{u}(k)$ are appropriate auxiliary variables. Now a method for selecting them is given to construct an augmented error system. Define

$$
\left\{\begin{array}{l}
\bar{x}(\infty)=x(\infty) \\
\bar{u}(\infty)=u(\infty)
\end{array}\right.
$$


If the controlled output of the nominal system (6) for system (1) can track the reference signal, there exist constant vectors $x(\infty)$ and $u(\infty)$ satisfying the equation of system (6). Thus letting $k$ go to infinity on both sides of equation (6), we obtain

$$
\left\{\begin{aligned}
x(\infty) & =A x(\infty)+B u(\infty), \\
r & =C x(\infty)
\end{aligned}\right.
$$

That is,

$$
\left[\begin{array}{cc}
A-I & B \\
C & 0
\end{array}\right]\left[\begin{array}{l}
x(\infty) \\
u(\infty)
\end{array}\right]=\left[\begin{array}{l}
0 \\
r
\end{array}\right]
$$

From Assumption 1, take

$$
\left[\begin{array}{l}
x(\infty) \\
u(\infty)
\end{array}\right]=\left[\begin{array}{cc}
A-I & B \\
C & 0
\end{array}\right]^{-1}\left[\begin{array}{l}
0 \\
r
\end{array}\right] .
$$

Thus, $x(\infty)$ and $u(\infty)$ are

$$
\left\{\begin{array}{l}
\bar{x}(\infty)=x(\infty)=S_{x} r(\infty) \\
\bar{u}(\infty)=u(\infty)=S_{u} r(\infty)
\end{array}\right.
$$

where

$$
S_{x}=\left[\begin{array}{ll}
I & 0
\end{array}\right]\left[\begin{array}{cc}
A-I & B \\
C & 0
\end{array}\right]^{-1}\left[\begin{array}{l}
0 \\
I
\end{array}\right], \quad S_{u}=\left[\begin{array}{ll}
0 & I
\end{array}\right]\left[\begin{array}{cc}
A-I & B \\
C & 0
\end{array}\right]^{-1}\left[\begin{array}{l}
0 \\
I
\end{array}\right] .
$$

The results of (10) are extended and the auxiliary variables are selected in the followings:

$$
\left\{\begin{array}{l}
\bar{x}(k)=S_{x} r(k), \\
\bar{u}(k)=S_{u} r(k) .
\end{array}\right.
$$

Remark 3: As described previously, the auxiliary variables $\bar{x}(k)$ and $\bar{u}(k)$ will be used to derive the augmented error system, in order to convert the tracking control problem into a state feedback $H_{\infty}$ control problem of the augmented error system. The difference method in $[2,5-6,17-20]$ will be found to be the equivalent to the choice of the auxiliary variables in (7) (i.e., $\bar{u}(k)=u(k-1), \bar{x}(k)=x(k-1)$ ). And the difference method in $[26,29]$ is the equivalent of $\bar{u}(k) \equiv u(\infty), \bar{x}(k) \equiv x(\infty)$. Since the uncertainties are time-varying and unknown in this paper, construction of the augmented error system through the usual difference method in [2, 5-6, 18-21] is impossible. If we use $\bar{u}(k)=u(\infty)$ and $\bar{x}(k)=x(\infty)$, the information on the nominal system cannot be fully used. Therefore, the method in this paper improves the previous methodologies. 


\section{Construction of the augmented error system}

In this section, an augmented error system which contains the error vector, state of the system, the future information and the integrator vector will be constructed and the robust $H_{\infty}$ control for the regulating system will be discussed. The state feedback controller with integral and preview actions achieving the robust tracking performance in terms of LMIs is obtained.

Define the error signal as

$$
e(k)=y(k)-r(k) \text {. }
$$

Combining (1), (7) and (11) gives

$$
\begin{aligned}
\tilde{x}(k+1)= & {[A+\Delta A] \tilde{x}(k)+[B+\Delta B] \tilde{u}(k)+[D+\Delta D] w(k)+[A+\Delta A] S_{x} r(k) } \\
& +[B+\Delta B] S_{u} r(k)-S_{x} r(k+1),
\end{aligned}
$$

and note that

$$
y(k)=C[x(k)-\bar{x}(k)]+C \bar{x}(k)=C \tilde{x}(k)+C S_{x} r(k) .
$$

Consequently, $\left\{\begin{aligned} \tilde{x}(k+1) & =[A+\Delta A] \tilde{x}(k)+[B+\Delta B] \tilde{u}(k)+[D+\Delta D] w(k)+[A+\Delta A] S_{x} r(k)+[B+\Delta B] S_{u} r(k)-S_{x} r(k+1), \\ y(k) & =C \tilde{x}(k)+C S_{x} r(k) .\end{aligned}\right.$

Furthermore, obviously have

$$
e(k)=C \tilde{x}(k)+\left[C S_{x}-I\right] r(k) .
$$

Using (15) and (16), one can obtain that

$$
\begin{aligned}
e(k+1)= & C\left\{[A+\Delta A] \tilde{x}(k)+[B+\Delta B] \tilde{u}(k)+[D+\Delta D] w(k)+[A+\Delta A] S_{x} r(k)\right. \\
& \left.+[B+\Delta B] S_{u} r(k)\right\}-r(k+1) .
\end{aligned}
$$

Define

$$
X_{R}(k)=\left[\begin{array}{c}
r(k) \\
r(k+1) \\
\vdots \\
\vdots \\
r\left(k+M_{R}\right)
\end{array}\right] \in R^{\left(M_{R}+1\right) q}, A_{R}=\left[\begin{array}{ccccc}
0 & I & & & 0 \\
0 & \ddots & \ddots & & \\
\vdots & & \ddots & \ddots & \\
0 & \cdots & \cdots & 0 & I \\
0 & \cdots & \cdots & 0 & 0
\end{array}\right] \in R^{\left[\left(M_{R}+1\right) q\right] \times\left(\left(M_{R}+1\right) q\right]}
$$

It follows from Assumption 2 that the equation can be obtained 


$$
X_{R}(k+1)=A_{R} X_{R}(k)
$$

Combining (15), (17), and (18) gives

$$
\left\{\begin{aligned}
\hat{x}(k+1) & =[\hat{A}+\Delta \hat{A}] \hat{x}(k)+[\hat{B}+\Delta \hat{B}] \tilde{u}(k)+[\hat{D}+\Delta \hat{D}] w(k), \\
e(k) & =\hat{C} \hat{x}(k),
\end{aligned}\right.
$$

where

$$
\begin{aligned}
& \hat{x}(k)=\left[\begin{array}{c}
e(k) \\
\tilde{x}(k) \\
X_{R}(k)
\end{array}\right], \hat{A}=\left[\begin{array}{ccc}
0 & C A & G_{p e} \\
0 & A & G_{p x} \\
0 & 0 & A_{R}
\end{array}\right], \Delta \hat{A}=\left[\begin{array}{ccc}
0 & C \Delta A & \Delta G_{p e} \\
0 & \Delta A & \Delta G_{p x} \\
0 & 0 & 0
\end{array}\right], \hat{B}=\left[\begin{array}{c}
C B \\
B \\
0
\end{array}\right], \\
& \Delta \hat{B}=\left[\begin{array}{c}
C \Delta B \\
\Delta B \\
0
\end{array}\right], \hat{D}=\left[\begin{array}{c}
C D \\
D \\
0
\end{array}\right], \Delta \hat{D}=\left[\begin{array}{c}
C \Delta D \\
\Delta D \\
0
\end{array}\right], \hat{C}=\left[\begin{array}{lll}
I & 0 & 0
\end{array}\right],
\end{aligned}
$$

and

$$
\begin{aligned}
& G_{p e}=\left[\begin{array}{lllll}
C\left(A S_{x}+B S_{u}\right) & -I & 0 & \cdots & 0
\end{array}\right], G_{p x}=\left[\begin{array}{llllll}
A S_{x}+B S_{u} & -S_{x} & 0 & \cdots & 0
\end{array}\right], \\
& \Delta G_{p x}=\left[\begin{array}{lllll}
\Delta A S_{x}+\Delta B S_{u} & 0 & \cdots & 0
\end{array}\right], \Delta G_{p e}=\left[\begin{array}{lllll}
C\left(\Delta A S_{x}+\Delta B S_{u}\right) & 0 & \cdots & 0
\end{array}\right]=C \Delta G_{p x},
\end{aligned}
$$

where $\hat{A}, \hat{B}, \hat{C}$ and $\hat{D}$ are constant matrices of the augmented error system, and $\Delta \hat{A}, \Delta \hat{B}$ and $\Delta \hat{D}$ are uncertain matrices.

Note that the main characteristic of system (19) contains the future information on the reference signal because the variable with future information is a part of the state variables. Furthermore, by introducing the previewable reference signal, instead of the difference signal, the signal itself is fully used.

Now considering the previous assumption about the uncertainty, the following will be obtained. 


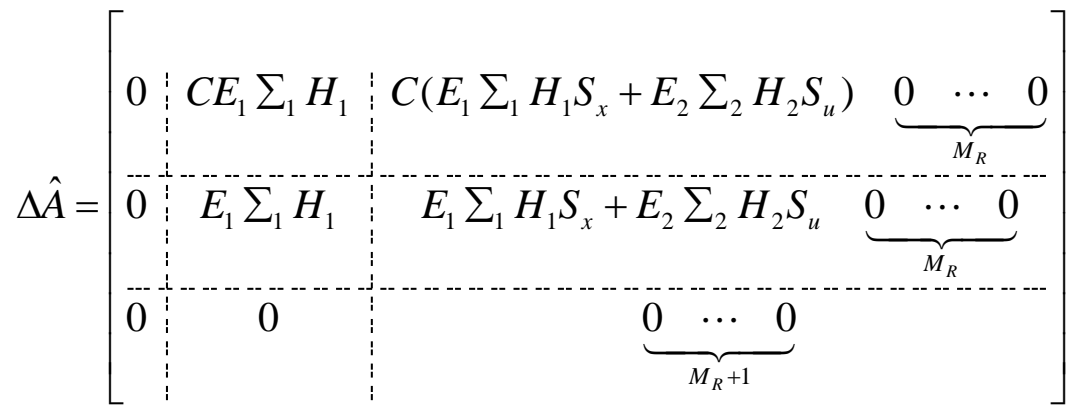

$$
\begin{aligned}
& =\left[\begin{array}{cc}
C E_{1} & C E_{2} \\
E_{1} & E_{2} \\
0 & 0
\end{array}\right]\left[\begin{array}{cc}
\Sigma_{1} & \\
& \Sigma_{2}
\end{array}\right]\left[\begin{array}{cccccc}
0 & H_{1} & H_{1} S_{x} & 0 & \cdots & 0 \\
0 & 0 & H_{2} S_{u} & 0 & \cdots & 0
\end{array}\right]=\hat{E}_{1} \hat{\Sigma}_{1} \hat{H}_{1} \text {, } \\
& \Delta \hat{B}=\left[\begin{array}{c}
C E_{2} \Sigma_{2} H_{2} \\
E_{2} \sum_{2} H_{2} \\
0
\end{array}\right]=\left[\begin{array}{c}
C E_{2} \\
E_{2} \\
0
\end{array}\right] \sum_{2} H_{2}=\hat{E}_{2} \hat{\Sigma}_{2} \hat{H}_{2} \\
& \Delta \hat{D}=\left[\begin{array}{c}
C E_{3} \Sigma_{3} H_{3} \\
E_{3} \Sigma_{3} H_{3} \\
0
\end{array}\right]=\left[\begin{array}{c}
C E_{3} \\
E_{3} \\
0
\end{array}\right] \sum_{3} H_{3}=\hat{E}_{3} \hat{\Sigma}_{3} \hat{H}_{3} .
\end{aligned}
$$

Note that the uncertain matrices still satisfy the matching conditions

$$
\hat{\Sigma}_{i}^{T} \hat{\Sigma}_{i} \leq I, \quad(i=1,2,3),
$$

and $\Delta \hat{A}, \Delta \hat{B}$ and $\Delta \hat{D}$ are norm bounded.

Note that the augmented system (19) does not contain the difference of $u(k)$. As a result, the obtained controller of system (19) based on the LMI approach does not include the integral of error $e(k)$. Therefore, an integrator will not appear in the final closed-loop system, which helps to eliminate the static error. Due to this reason the discrete integrator is introduced and defined by

$$
v(k+1)=v(k)+e(k)
$$

namely,

$$
v(k)=\sum_{j=0}^{k-1} e(j)+v(0)
$$

where $v(0)$ can be assigned as needed. In general, we take $v(0)=0$.

Define $X(k)=\left[\begin{array}{l}\hat{x}(k) \\ v(k)\end{array}\right]$ again, combining (19) and (24), we get 


$$
\left\{\begin{aligned}
X(k+1) & =[F+\Delta F] X(k)+[G+\Delta G] \tilde{u}(k)+[H+\Delta H] w(k), \\
e(k) & =U X(k)
\end{aligned}\right.
$$

where

$$
\begin{aligned}
& F=\left[\begin{array}{cc}
\hat{A} & 0 \\
C_{E} & I
\end{array}\right], \Delta F=\left[\begin{array}{cc}
\Delta \hat{A} & 0 \\
0 & 0
\end{array}\right], \quad G=\left[\begin{array}{c}
\hat{B} \\
0
\end{array}\right], \Delta G=\left[\begin{array}{c}
\Delta \hat{B} \\
0
\end{array}\right], H=\left[\begin{array}{c}
\hat{D} \\
0
\end{array}\right], \Delta H=\left[\begin{array}{c}
\Delta \hat{D} \\
0
\end{array}\right], \\
& C_{E}=\left[\begin{array}{lll}
I & 0 & 0
\end{array}\right], U=\left[\begin{array}{ll}
\hat{C} & 0
\end{array}\right] .
\end{aligned}
$$

It can be seen from (20), (21), and (22) that the uncertain matrices in system (25) can be expressed as

$$
\begin{aligned}
& \Delta F=\left[\begin{array}{cc}
\hat{E}_{1} \hat{\Sigma}_{1} \hat{H}_{1} & 0 \\
0 & 0
\end{array}\right]=\left[\begin{array}{c}
\hat{E}_{1} \\
0
\end{array}\right] \hat{\Sigma}_{1}\left[\hat{H}_{1} \quad 0\right]=E_{11} \sum_{11} H_{11}, \\
& \Delta G=\left[\begin{array}{c}
\hat{E}_{2} \hat{\Sigma}_{2} \hat{H}_{2} \\
0
\end{array}\right]=\left[\begin{array}{c}
\hat{E}_{2} \\
0
\end{array}\right] \hat{\Sigma}_{2} \hat{H}_{2}=E_{22} \Sigma_{22} H_{22}, \\
& \Delta H=\left[\begin{array}{c}
\hat{E}_{3} \hat{\Sigma}_{3} \hat{H}_{3} \\
0
\end{array}\right]=\left[\begin{array}{c}
\hat{E}_{3} \\
0
\end{array}\right] \hat{\Sigma}_{3} \hat{H}_{3}=E_{33} \Sigma_{33} H_{33} .
\end{aligned}
$$

(23) leads to the fact that the uncertain matrices still satisfy

$$
\sum_{i i}^{T} \sum_{i i} \leq I, \quad(i=1,2,3) .
$$

System (25) is the derived augmented error system. Because $e(k)$ is the part of the state vector $X(k)$, if a feedback control is designed to guarantee the asymptotic stability of the closed-loop system, then the output $y(k)$ of the closed-loop system of system (1) can track the desired tracking signal $r(k)$ with no static error.

\section{Design of a preview controller}

Consider the nominal system of system $(25)[\tilde{u}(t)=0]$

$$
\left\{\begin{aligned}
X(k+1) & =F X(k)+H w(k), \\
e(k) & =U X(k) .
\end{aligned}\right.
$$

Theorem 1: For prescribed $\gamma>0$, system (30) is asymptotically stable with prescribed $H_{\infty}$ performance $\gamma$, that is, $\|e(k)\|_{2} \leq \gamma\|w(k)\|_{2}$ if there exist matrix $P>0$ and matrices $T_{1}, T_{2}$ with appropriate dimensions such that 


$$
\Omega=\left[\begin{array}{ccc}
-P+U^{T} U-T_{1} F-F^{T} T_{1}^{T} & -T_{1} H & T_{1}-F^{T} T_{2}^{T} \\
-H^{T} T_{1}^{T} & -\gamma^{2} I & -H^{T} T_{2}^{T} \\
T_{1}^{T}-T_{2} F & -T_{2} H & P+T_{2}+T_{2}^{T}
\end{array}\right]<0 .
$$

Proof: Construct the positive definite Lyapunov function according to the matrix $P$ in $(31)$

$$
V(X(k))=X(k)^{T} P X(k) .
$$

From (30), for any matrices $T_{1}$ and $T_{2}$ with appropriate dimensions the following equation holds:

$$
2\left[X(k)^{T} T_{1}+X(k+1)^{T} T_{2}\right][X(k+1)-F X(k)-H w(k)]=0 .
$$

Calculating the difference of $V(X(k))$ along the trajectory of system (30) and adding (32) to it yields

$$
\left.\begin{array}{rl}
\Delta V+e(k)^{T} e(k)-\gamma^{2} w(k)^{T} w(k) & =X(k+1)^{T} P X(k+1)-X(k)^{T} P X(k) \\
+e(k)^{T} e(k)-\gamma^{2} w(k)^{T} w(k)+2\left[X(k)^{T} T_{1}+X(k+1)^{T} T_{2}\right.
\end{array}\right]\left[\begin{array}{ll}
X(k+1)-F X(k)-H w(K)] \\
=
\end{array}\right.
$$

Since $\Omega<0$, then $\Delta V+e(k)^{T} e(k)-\gamma w(k)^{T} w(k)<0$ for any $X(k) \neq 0$. And the initial condition $X(0)=0$ implies that $\|e(k)\|_{2} \leq \gamma\|w(k)\|_{2}, w(k) \in l_{2}[30-32]$.

Remark 4: The introduction of free weighting matrices leads to the freedom of some parameters in Theorem 1. Therefore, the conservatism of the solutions can be reduced. In fact, by setting $T_{1}=0, T_{2}=-P$, this condition of Theorem 1 is transformed into Lemma 6 in [30]. Actually, based on Lemma 6 in [30], the robust $H_{\infty}$ control problems were considered by references [31-33]. Therefore, the obtained results may generalize the relevant results in [31-33].

Now, the purpose of the preview control is to design a state feedback controller for system (25) in the form of

$$
\tilde{u}(k)=K X(k),
$$

such that it makes the closed-loop system of system (25) asymptotically stable. We will give the controller gain matrix in (33) by using the relevant theory and LMI approach. 
From (25) and (33), the following will be obtained:

$$
\left\{\begin{aligned}
X(k+1) & =[F+\Delta F+(G+\Delta G) K] X(k)+[H+\Delta H] w(k), \\
e(k) & =U X(k) .
\end{aligned}\right.
$$

Theorem 2: Given a scalar $\gamma>0$, a adjustable scalar $\alpha$ and a adjustable scalar $\beta \in(0,2)$, if there exist matrices $X>0, Y$ and constant scalars $\varepsilon_{i}>0,(i=1,2,3)$ such that the following LMI holds:

$$
\left[\begin{array}{ccccccc}
-\alpha^{2} X-\operatorname{sym}(\alpha(F X+G Y))+\Upsilon & * & * & * & * & * & * \\
-H^{T} & -\gamma^{2} I & * & * & * & * & * \\
-\beta X-\alpha(F X+G Y)+\Upsilon & -H & \left(\beta^{2}-2 \beta\right) X+\Upsilon & * & * & * & * \\
\alpha H_{11} X & 0 & 0 & -\varepsilon_{1} I & * & * & * \\
\alpha H_{22} Y & 0 & 0 & 0 & -\varepsilon_{2} I & * & * \\
0 & H_{33} & 0 & 0 & 0 & -\varepsilon_{3} I & * \\
\alpha U X & 0 & 0 & 0 & 0 & 0 & -I
\end{array}\right]<0,
$$

where $\Upsilon=\varepsilon_{1} E_{11} E_{11}{ }^{T}+\varepsilon_{2} E_{22} E_{22}{ }^{T}+\varepsilon_{3} E_{33} E_{33}{ }^{T}$, then system (34) is robustly stabilizable via (33) and the control gain matrix is given by $K=Y X^{-1}$.

Proof: For the closed-loop system, it can be proved that if (35) is established, then the conditions of Theorem 1 are established: thus, from Theorem 1, Theorem 2 holds.

For the system (34), it follows from Theorem 1 that given a scalar $\gamma>0$, if there exist $P>0, T_{1}$ and $T_{2}$ with appropriate dimensions satisfying

$$
\left[\begin{array}{ccc}
-P+U^{T} U-T_{1} \Theta-\Theta^{T} T_{1}^{T} & -T_{1}(H+\Delta H) & T_{1}-\Theta^{T} T_{2}^{T} \\
-(H+\Delta H)^{T} T_{1}^{T} & -\gamma^{2} I & -(H+\Delta H)^{T} T_{2}^{T} \\
T_{1}^{T}-T_{2} \Theta & -T_{2}(H+\Delta H) & P+T_{2}+T_{2}^{T}
\end{array}\right]<0,
$$

where $\Theta=F+\Delta F+(G+\Delta G) K$, then the closed-loop system (34) is asymptotically stable and satisfies $\|e(k)\|_{2} \leq \gamma\|w(k)\|_{2}$.

$\Psi \quad$ will be used to denote the left matrix of (36) for simplicity in the following (37), and separated the uncertainties from $\Psi$. Then, together with (26)-(28), the following will be obtained:

$$
\Psi=\Phi+\Xi_{1} \Xi_{2} \Xi_{3}+\Xi_{3}{ }^{T} \Xi_{2}{ }^{T} \Xi_{1}{ }^{T},
$$


where

$$
\begin{aligned}
& \Phi=\left[\begin{array}{ccc}
-P+U^{T} U-T_{1} F-F^{T} T_{1}^{T} & -T_{1} H & T_{1}-F^{T} T_{2}^{T} \\
-H^{T} T_{1}^{T} & -\gamma^{2} I & -H^{T} T_{2}^{T} \\
T_{1}^{T}-T_{2} F & -T_{2} H & P+T_{2}+T_{2}^{T}
\end{array}\right], \\
& \Xi_{1}=\left[\begin{array}{ccc}
-T_{1} E_{11} & -T_{1} E_{22} & -T_{1} E_{33} \\
0 & 0 & 0 \\
-T_{2} E_{11} & -T_{2} E_{22} & -T_{2} E_{33}
\end{array}\right], \Xi_{2}=\left[\begin{array}{ccc}
\Sigma_{11} & \\
& \sum_{22} \\
& & \Sigma_{44}
\end{array}\right], \Xi_{3}=\left[\begin{array}{ccc}
H_{11} & 0 & 0 \\
H_{22} K & 0 & 0 \\
0 & H_{33} & 0
\end{array}\right] .
\end{aligned}
$$

It follows from (29) that $\Xi_{2}{ }^{T} \Xi_{2} \leq I$. Lemma 2 is presented to be applied to the latter two items of the left-hand side matrix in (37), and letting $\Lambda=\left[\begin{array}{ccc}\varepsilon_{1} I & 0 & 0 \\ 0 & \varepsilon_{2} I & 0 \\ 0 & 0 & \varepsilon_{3} I\end{array}\right]$, $\varepsilon_{1}>0, \varepsilon_{2}>0, \varepsilon_{3}>0$, we obtain

$$
\Psi<\Phi+\Xi_{1} \Lambda \Xi_{1}^{T}+\Xi_{3}^{T} \Lambda^{-1} \Xi_{3}
$$

Hence, if there exist $\varepsilon_{1}>0, \varepsilon_{2}>0, \varepsilon_{3}>0$ such that

$$
\Phi+\Xi_{1} \Lambda \Xi_{1}^{T}+\Xi_{3}^{T} \Lambda^{-1} \Xi_{3}<0,
$$

then $\Psi<0$, thus, the condition of Theorem 1 is satisfied. Now the necessary and sufficient condition for (38) is discussed. According to the Lemma 1, it follows that (38) is equivalent to

$$
\left[\begin{array}{ccccccc}
\Lambda_{1} & -T_{1} H & \Lambda_{2}{ }^{T} & H_{11}{ }^{T} & K^{T} H_{22}{ }^{T} & 0 & U^{T} \\
-H^{T} T_{1}^{T} & -\gamma^{2} I & -H^{T} T_{2}^{T} & 0 & 0 & H_{33}{ }^{T} & 0 \\
\Lambda_{2} & -T_{2} H & \Lambda_{3} & 0 & 0 & 0 & 0 \\
H_{11} & 0 & 0 & -\varepsilon_{1} I & 0 & 0 & 0 \\
H_{22} K & 0 & 0 & 0 & -\varepsilon_{2} I & 0 & 0 \\
0 & H_{33} & 0 & 0 & 0 & -\varepsilon_{3} I & 0 \\
U & 0 & 0 & 0 & 0 & 0 & -I
\end{array}\right]<0
$$

where

$$
\begin{aligned}
& \Lambda_{1}=-P-T_{1}(F+G K)-(F+G K)^{T} T_{1}^{T}+\varepsilon_{1} T_{1} E_{11} E_{11}{ }^{T} T_{1}^{T}+\varepsilon_{2} T_{1} E_{22} E_{22}{ }^{T} T_{1}^{T}+\varepsilon_{3} T_{1} E_{33} E_{33}{ }^{T} T_{1}^{T}, \\
& \Lambda_{2}=T_{1}^{T}-T_{2} F+\varepsilon_{1} T_{2} E_{11} E_{11}{ }^{T} T_{1}^{T}+\varepsilon_{2} T_{2} E_{22} E_{22}{ }^{T} T_{1}^{T}+\varepsilon_{3} T_{2} E_{33} E_{33}{ }^{T} T_{1}^{T}, \\
& \Lambda_{3}=P+T_{2}+T_{2}^{T}+\varepsilon_{1} T_{2} E_{11} E_{11}{ }^{T} T_{2}^{T}+\varepsilon_{2} T_{2} E_{22} E_{22}{ }^{T} T_{2}^{T}+\varepsilon_{3} T_{2} E_{33} E_{33}{ }^{T} T_{2}^{T} .
\end{aligned}
$$

The parameter adjustment method [34-35] is proposed, setting 


$$
T_{1}=a P, T_{2}=-b P
$$

where $a$ and $b$ are adjustable scalars. Then, by performing congruence transformations by an invertible symmetric matrix $\operatorname{diag}\left\{T_{1}^{-1}, I, T_{2}^{-1}, I\right\}$ to (39) and denoting $P^{-1}=X, K X=Y, \alpha=\frac{1}{a}, \quad \beta=\frac{1}{b}$, it arrives at the condition in Theorem 2, or equivalently (35) holds.

It has been proved that when $T_{1}$ and $T_{2}$ are given by (40) and the adjustable scalars are selected properly, if (35) holds, then (39) and thereby (36) hold. Thus Theorem 2 is proved.

Remark 5: if (35) holds, then $\left(\beta^{2}-2 \beta\right) X+\Upsilon<0$. And from the structure of $\Upsilon$, we obtain $\Upsilon \geq 0$, thereby $\left(\beta^{2}-2 \beta\right) X<0$, and combining $X>0$ yields $0<\beta<2$.

When $\gamma$ is considered as a decision variable which should be minimized. The following will be obtained.

Theorem 3: Consider the system (25) with (33). If the following optimization problem:

$$
\underset{\bar{\gamma}, \varepsilon_{1}, \varepsilon_{2}, \varepsilon_{3}, \alpha, \beta, X, Y}{\min }(\bar{\gamma})
$$

s.t.

$$
\left[\begin{array}{ccccccc}
-\alpha^{2} X-\operatorname{sym}(\alpha(F X+G Y))+\Upsilon & * & * & * & * & * & * \\
-H^{T} & -\bar{\gamma} & * & * & * & * & * \\
-\beta X-\alpha(F X+G Y)+\Upsilon & -H & \left(\beta^{2}-2 \beta\right) X+\Upsilon & * & * & * & * \\
\alpha H_{11} X & 0 & 0 & -\varepsilon_{1} I & * & * & * \\
\alpha H_{22} Y & 0 & 0 & 0 & -\varepsilon_{2} I & * & * \\
0 & H_{33} & 0 & 0 & 0 & -\varepsilon_{3} I & * \\
\alpha U X & 0 & 0 & 0 & 0 & 0 & -I
\end{array}\right]<0
$$

has a solution $\bar{\gamma}>0, \varepsilon_{i}>0,(i=1,2,3), X>0$ and $Y$. Then the system (34) is robustly stabilizable with disturbance attenuation $\gamma=\sqrt{\bar{\gamma}}$ by (33) and the control gain matrix is given by $K=Y X^{-1}$.

Now we discuss the control input of system (1). 
When Assumption 1-Assumption 3 are satisfied, the control input (33) of system (25) is obtained. The gain matrix $K$ will be decomposed into:

$$
K=\left[\begin{array}{lllllll}
K_{e} & K_{x} & K_{R}(0) & K_{R}(1) & \cdots & K_{R}\left(M_{R}\right) & K_{v}
\end{array}\right] .
$$

Based on (33) and (42), the following will be obtained:

$$
\tilde{u}(k)=K_{e} e(k)+K_{x} \tilde{x}(k)+\sum_{i=0}^{M_{R}} K_{R}(i) r(k+i)+K_{v}\left(\sum_{s=0}^{k-1} e(s)+v(0)\right) .
$$

The main theorem of the paper is presented by synthesizing the above theorems.

Theorem 4: Suppose that Assumption 1-Assumption 3 are satisfied. The controller of system (1) can be taken as

$$
u(k)=K_{e} e(k)+K_{x} x(k)+\sum_{i=0}^{M_{R}} K_{R}(i) r(k+i)+K_{v}\left(\sum_{s=0}^{k-1} e(s)+v(0)\right)+\left(S_{u}-K_{x} S_{x}\right) r(k),
$$

where $K=Y X^{-1}, X>0$ and $Y$ can be determined by (41), and (42) determines the relationship between $K_{e}, K_{x}, K_{R}(0), \cdots, K_{R}\left(M_{R}\right), K_{v}$ and $K ; S_{x}$ and $S_{u}$ are solved by (10). And under this controller, the closed-loop system of system (1) can achieve good tracking of the reference signal.

Proof: When Assumption 1-Assumption 3 are satisfied, the augmented error system (25) can be derived. If the LMI optimization problem determined by (41) in Theorem 3 has a solution $\left(\varepsilon_{1}, \varepsilon_{2}, \varepsilon_{3}, \alpha, \beta, \bar{\gamma}, X, Y\right)$, by Theorem 3 , one gets

$$
\tilde{u}(k)=Y X^{-1} X(k)=K X(k)
$$

the gain matrix $K$ in decomposed into:

$$
K=\left[\begin{array}{lllllll}
K_{e} & K_{x} & K_{R}(0) & K_{R}(1) & \cdots & K_{R}\left(M_{R}\right) & K_{v}
\end{array}\right]=Y X^{-1},
$$

then (44) can be written as

$$
\tilde{u}(k)=K_{e} e(k)+K_{x} \tilde{x}(k)+\sum_{i=0}^{M_{R}} K_{R}(i) r(k+i)+K_{v}\left(\sum_{s=0}^{k-1} e(s)+v(0)\right) .
$$

By (7) and (11), the following will be derived:

$$
u(k)=K_{e} e(k)+K_{x} x(k)+\sum_{i=0}^{M_{R}} K_{R}(i) r(k+i)+K_{v}\left(\sum_{s=0}^{k-1} e(s)+v(0)\right)+\left(S_{u}-K_{x} S_{x}\right) r(k) .
$$

Therefore, theorem 4 holds.

In light of the above equation, it is clear that the preview controller of system (1) consists of five parts. The first part is tracking error compensation, the second part is the 
state feedback, the third part is reference preview feed-forward compensation, the fourth part is the integration of the tracking error term, and the last one is the compensation by the initial and final values.

Remark 6: An augmented plant including previewed information is constructed by using the error system method in preview control theory and the discrete-time lifting technique. Then the robust stability for the augmented error system of uncertain system is analyzed and a preview controller is designed by combining the robust control theory and LMI approach. Based on the key ideas above, the design method of the robust preview controller is presented. Our results extend some recent results of references [11-21].

\section{Design of state observer}

If the state variables in the original system cannot be measured as feedback state variables, equivalently the partial variables $\tilde{x}(k)$ of $X(k)$ in system (25) cannot be available. A state observer to reconstruct $\tilde{x}(k)$ can be constructed. Due to this reason, each part of the vector $X(k)$ is rearranged and system (25) is rewritten as $\left[\begin{array}{c}e(k+1) \\ X_{R}(k+1) \\ v(k+1) \\ \hdashline \tilde{x}(k+1)\end{array}\right]=\left[\begin{array}{ccc:c}0 & G_{p e}+\Delta G_{p e} & 0 & C[A+\Delta A] \\ 0 & A_{R} & 0 & 0 \\ I & 0 & I & 0 \\ \hdashline 0 & G_{p x}+\Delta G_{p x} & 0 & A+\Delta A\end{array}\right]\left[\begin{array}{c}e(k) \\ X_{R}(k) \\ v(k) \\ \hdashline \tilde{x}(k)\end{array}\right]+\left[\begin{array}{c}C[B+\Delta B] \\ 0 \\ 0 \\ \hdashline B+\Delta B\end{array}\right] \tilde{u}(k)+\left[\begin{array}{c}C[D+\Delta D] \\ 0 \\ 0 \\ \hdashline D+\Delta D\end{array}\right] w(k)$.

Considering the observation equation of system (1), the predictability of the reference signal, and the introduction of the integrator, the observation equation of system (45) can be taken as

$$
Z(k)=C_{Z} \hat{X}(k)
$$

where

$$
C_{Z}=\left[\begin{array}{ccc:c}
I_{q \times q} & 0 & 0 & 0 \\
0 & I_{\left[\left(M_{R}+1\right) q\right] \times\left[\left(M_{R}+1\right) q\right]} & 0 & 0 \\
0 & 0 & I_{q \times q} & 0
\end{array}\right], \hat{X}(k)=\left[\begin{array}{c}
e(k) \\
X_{R}(k) \\
v(k) \\
\hdashline \tilde{x}(k)
\end{array}\right]
$$


If the nominal system of system (45) is observable, there exists a reduced-order observer with the related theory of a discrete-time observer [36, 37]. Therefore, in the following, the reduced-order observer is constructed by

$$
\begin{aligned}
W(k+1) & =\left[A+L\left(\begin{array}{c}
C A \\
0 \\
0
\end{array}\right)\right] W(k)+\left[B+L\left(\begin{array}{c}
C B \\
0 \\
0
\end{array}\right)\right] \tilde{u}(k)+\left[\begin{array}{lll}
0 & G_{p x} & 0
\end{array}\right)+L\left(\begin{array}{ccc}
0 & G_{p e} & 0 \\
0 & A_{R} & 0 \\
I & 0 & I
\end{array}\right) \\
& \left.-\left[A+L\left(\begin{array}{c}
C A \\
0 \\
0
\end{array}\right)\right] L\right] Z(k), \\
\hat{\tilde{x}}(k)= & W(k)-L Z(k),
\end{aligned}
$$

where $L \in R^{n \times\left[q+\left(M_{R}+1\right) q+q\right]}$ is the observer gain matrix that makes the eigenvalues of $A+L\left(\begin{array}{c}C A \\ 0 \\ 0\end{array}\right)$ be located in the unit circle.

Note that if we let $L=\left[\begin{array}{lll}L_{1} & L_{2} & L_{3}\end{array}\right]$, where $L_{1} \in R^{n \times q}, L_{2} \in R^{n \times\left[q\left(M_{R}+1\right)\right]}, \quad L_{3} \in R^{n \times q}$, then $A+L\left(\begin{array}{c}C A \\ 0 \\ 0\end{array}\right)=A+L_{1} C A$; as a result, we only need place the poles of the $n$-order matrix and the observer is designed without using $H_{\infty}$ control.

Remark 7: The designed observer (47) for the augmented error system (45) is a reduced-order observer, while it is full-order for system (15). As with the literature [38], the designed reduced-order observer for the augmented error system and the designed full observer for the original system are totally different. The output equation of system (45) contains the information of the previewable reference signal through reformulation of the output equation. Therefore, the previewable reference signal can be fully utilized in the design process. In addition, in designing the observer, the eigenvalues of $A+L_{1} C A$ must be located in the unit circle and $L_{2}, L_{3}$ can be selected as needed without increasing the difficulty of the eigenvalue configuration. And in fact, when an observer is designed for system (15), $L$ is selected to ensure that $A+L C$ is a stable matrix. 
Based on the above analysis, the condition of existence of an observer (47) for the nominal system of system (45) is that the nominal system is observable. In the following, the PBH rank test [2] will be employed to prove the observability of the nominal system of augmented error system (45). For the sake of notational convenience, we denote the state matrix of the nominal system of system (45) with $\Gamma$.

Lemma 3: $\left(C_{Z}, \Gamma\right)$ is observable if and only if $(C, A)$ is observable and $A$ is invertible.

Proof: By the $\mathrm{PBH}$ criteria, $\left(C_{Z}, \Gamma\right)$ is observable if and only if the matrix

$$
\left[\begin{array}{c}
\Gamma-s I \\
C_{Z}
\end{array}\right]
$$

has column full rank for any complex $s$.

From the expression of $\Gamma$ and $C_{Z}$, it can be seen that

$$
\left[\begin{array}{c}
\Gamma-s I \\
C_{Z}
\end{array}\right]=\left[\begin{array}{cccc}
-s I & G_{p e} & 0 & C A \\
0 & A_{R}-s I & 0 & 0 \\
I & 0 & (1-s) I & 0 \\
0 & G_{p x} & 0 & A-s I \\
\hdashline I_{q} & 0 & 0 & 0 \\
0 & I_{\left(M_{R}+1\right) q} & 0 & 0 \\
0 & 0 & I_{q} & 0
\end{array}\right] .
$$

$s I$ is nonsingular for any complex $s$ satisfying $|s| \neq 0$. By elementary transformation of the matrix, we have

$$
\left[\begin{array}{c}
\Gamma-s I \\
C_{Z}
\end{array}\right] \rightarrow\left[\begin{array}{cccc}
0 & 0 & 0 & C A \\
0 & 0 & 0 & 0 \\
0 & 0 & 0 & 0 \\
0 & 0 & 0 & A-s I \\
\hdashline I_{q} & 0 & 0 & 0 \\
0 & I_{\left(M_{R}+1\right) q} & 0 & 0 \\
0 & 0 & I_{q} & 0
\end{array}\right] \rightarrow\left[\begin{array}{cccc}
0 & 0 & 0 & -s C \\
0 & 0 & 0 & 0 \\
0 & 0 & 0 & 0 \\
0 & 0 & 0 & A-s I \\
\hdashline I_{q} & 0 & 0 & 0 \\
0 & I_{\left(M_{R}+1\right) q} & 0 & 0 \\
0 & 0 & I_{q} & 0
\end{array}\right] .
$$

Thus the matrix $\left[\begin{array}{c}\Gamma-s I \\ C_{Z}\end{array}\right]$ has full column rank if and only if $\left[\begin{array}{c}A-s I \\ C\end{array}\right]$ is of full column rank. 
When $s=0$, one gets from elementary transformation of matrix

$$
\left[\begin{array}{c}
\Gamma-s I \\
C_{Z}
\end{array}\right]_{s=0} \rightarrow\left[\begin{array}{cccc}
0 & 0 & 0 & C A \\
0 & 0 & 0 & 0 \\
0 & 0 & 0 & 0 \\
0 & 0 & 0 & A \\
\hdashline I_{q} & 0 & 0 & 0 \\
0 & I_{\left(M_{R}+1\right) q} & 0 & 0 \\
0 & 0 & I_{q} & 0
\end{array}\right] \rightarrow\left[\begin{array}{cccc}
0 & 0 & 0 & 0 \\
0 & 0 & 0 & 0 \\
0 & 0 & 0 & 0 \\
0 & 0 & 0 & A \\
\hdashline I_{q} & 0 & 0 & 0 \\
0 & I_{\left(M_{R}+1\right) q} & 0 & 0 \\
0 & 0 & I_{q} & 0
\end{array}\right] .
$$

Thus the matrix $\left[\begin{array}{c}\Gamma-s I \\ C_{Z}\end{array}\right]_{s=0}$ is of full column rank if and only if $A$ has full column rank, that is, $A$ is invertible.

Lemma 3 holds.

\section{Numerical example}

$$
\begin{aligned}
& \text { In system (1), let } A=\left[\begin{array}{cccc}
1.4 & 0.5 & 0.2 & -0.1 \\
0 & 0.90 & 0 & 0.19 \\
-0.24 & 0.24 & 0.97 & 0.02 \\
0.24 & -0.24 & 0.02 & 0.9
\end{array}\right], B=\left[\begin{array}{c}
-0.2 \\
0 \\
-0.2 \\
-0.03
\end{array}\right], \quad C=\left[\begin{array}{llll}
0 & 1 & 0 & 0
\end{array}\right] \text {, } \\
& D=\left[\begin{array}{c}
0.2 \\
0 \\
0.3 \\
-0.1
\end{array}\right], E_{1}=\left[\begin{array}{cccc}
0.2 & 0 & 0 & 0 \\
0 & 0.2 & 0 & 0 \\
0 & 0 & -0.2 & 0 \\
0 & 0 & 0 & 0.2
\end{array}\right], H_{1}=\left[\begin{array}{cccc}
0.02 & 0 & 0 & 0 \\
0 & 0.02 & 0 & 0 \\
0 & 0 & 0.02 & 0 \\
0 & 0 & 0 & 0
\end{array}\right] \text {, } \\
& \Sigma_{1}=\left[\begin{array}{cccc}
a_{1} & 0 & 0 & 0 \\
0 & 0.5 \cos (0.3 \pi k)+a_{2} & 0 & 0 \\
0 & 0 & 0.4 \sin (0.5 \pi k)+a_{3} & 0 \\
0 & 0 & 0 & 0.1
\end{array}\right], E_{2}=\left[\begin{array}{cccc}
0.2 & 0 & 0 & 0 \\
0 & 0.2 & 0 & 0 \\
0 & 0 & 0.2 & 0 \\
0 & 0 & 0 & 0.2
\end{array}\right] \text {, } \\
& H_{2}=\left[\begin{array}{c}
0.02 \\
-0.01 \\
0 \\
0.01
\end{array}\right], \Sigma_{2}=\left[\begin{array}{cccc}
a_{4} & 0 & 0.1 \cos (0.3 \pi k) & 0 \\
0 & 0.1 \cos (0.3 \pi k)+a_{5} & 0 & 0 \\
0 & 0 & 0.02 & 0 \\
0 & 0 & 0 & -0.02
\end{array}\right] \text {, }
\end{aligned}
$$




$$
E_{3}=\left[\begin{array}{cccc}
0.2 & 0 & 0 & 0 \\
0 & 0.2 & 0 & 0 \\
0 & 0 & 0.2 & 0 \\
0 & 0 & 0 & 0.2
\end{array}\right], H_{3}=\left[\begin{array}{c}
0.02 \\
-0.02 \\
0.01 \\
-0.01
\end{array}\right], \quad \sum_{3}=0
$$

Through verifying, $\left[\begin{array}{cc}A-I & B \\ C & 0\end{array}\right]$ is invertible, and $(C, A)$ is detectable and $\Sigma_{1}$, $\Sigma_{2}$ and $\sum_{3}$ satisfy (5) for all $k$. Therefore, the system satisfies the basic assumptions.

The exogenous disturbance is taken as

$$
w(k)=\left\{\begin{array}{cc}
1.5, & 15 \leq k \leq 60, \\
0, & \text { other }
\end{array}\right.
$$

The reference signal is taken as

$$
r(k)= \begin{cases}2, & k \geq 30 \\ 0, & k<30\end{cases}
$$

Simulations for three situations are performed in the following. The preview lengths of the reference signal are $M_{R}=7, M_{R}=2$ with no preview (i.e., $M_{R}=0$ ). According to Theorem 3, we use the LMI toolbox of MATLAB to solve matrix variables $X, Y$ and $\bar{\gamma}$ in LMI (41); the adjustable variables are selected as $\alpha=10, \beta=0.5$, and then the gain matrix $K=Y X^{-1}$ and $\gamma_{\min }=0.98126$ in (41) are obtained naturally.

When $M_{R}=2$, the following will be obtained:

$$
\begin{aligned}
K= & {\left[\begin{array}{lllll:ll}
7.25749 & 7.79562 & 30.05994 & 0.70340 & 27.47107 & 53.73314 & -7.62783 \\
& -8.18856 & 7.25749
\end{array}\right], }
\end{aligned}
$$

and

$$
\begin{aligned}
& K_{e}=7.25749, \quad K_{x}=\left[\begin{array}{llll}
7.79562 & 30.05994 & 0.70340 & 27.47107
\end{array}\right] \text {, } \\
& K_{R}=\left[\begin{array}{lll}
53.73314 & -7.62783 & -8.18856
\end{array}\right] \text {, } \\
& K_{v}=7.25749 \text {. }
\end{aligned}
$$

When $M_{R}=7, K$ will obtained as follows:

$$
K=\left[\begin{array}{llll}
K_{e} & K_{x} & K_{R} & K_{v}
\end{array}\right] \in R^{1 \times 14},
$$$$
K_{e}=7.25749
$$ 


$$
\begin{aligned}
& K_{x}=\left[\begin{array}{llll}
7.79562 & 30.05994 & 0.70340 & 27.47107
\end{array}\right], \\
& K_{R}=\left[\begin{array}{llllllll}
53.73314 & -7.62783 & -8.18856 & -8.68922 & -8.89846 & -8.69581 & -8.05784 & -7.04074
\end{array}\right] \text {, } \\
& K_{v}=7.25749 \text {. } \\
& \text { When } M_{R}=0, K \text { will be: } \\
& K=\left[\begin{array}{lll}
K_{e} & K_{x} & K_{v}
\end{array}\right] \\
& =\left[\begin{array}{l:llll:l}
7.32823 & 7.84756 & 31.05525 & 0.67990 & 27.75698 & 7.34080
\end{array}\right] \text {. }
\end{aligned}
$$

Take the allowed initial states are assumed as $x(0)=\left[\begin{array}{llll}0 & 0 & 0 & 0\end{array}\right]^{T}$ and $v(0)=0$.

In order to reflect the uncertainty, the uncertain parameters $a_{i}(i=1,2,3,4,5)$, with the absolute values no more than 0.5 , are taken as random numbers. Note that if the selecting method for the auxiliary variables $\bar{x}(k)$ and $\bar{u}(k)$ in [39] is adopted, then the initial conditions should be decided based on different situations. The proposed method can avoid the limitation on initial conditions.

The output curves of system (1) are depicted in Figure 1 and Figure 2 plots the tracking error. We can see that the output $y(k)$ can all track the reference signal accurately with the preview length of the reference signal $M_{R}=7, M_{R}=2$, and no preview, respectively. From figures1-3, we can find that the preview controller provides better performance than the controller with no preview compensation. And the preview action can reduce the tracking error and the input peak, and accelerate the speed of the output response tracking the reference signal. This is exactly how preview control achieves its goal. 


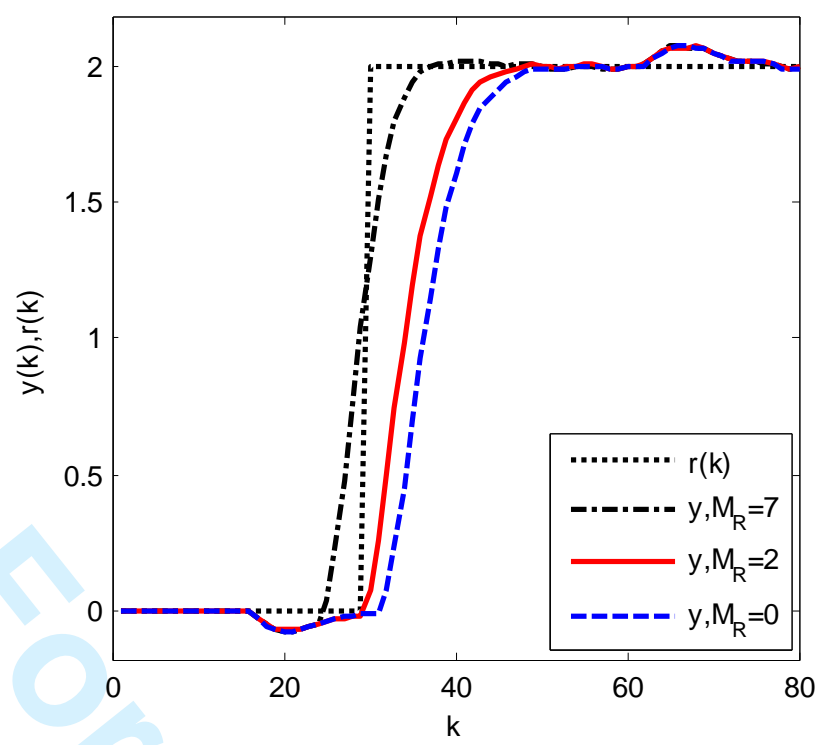

Figure 1. The output response of uncertain system to step signal.

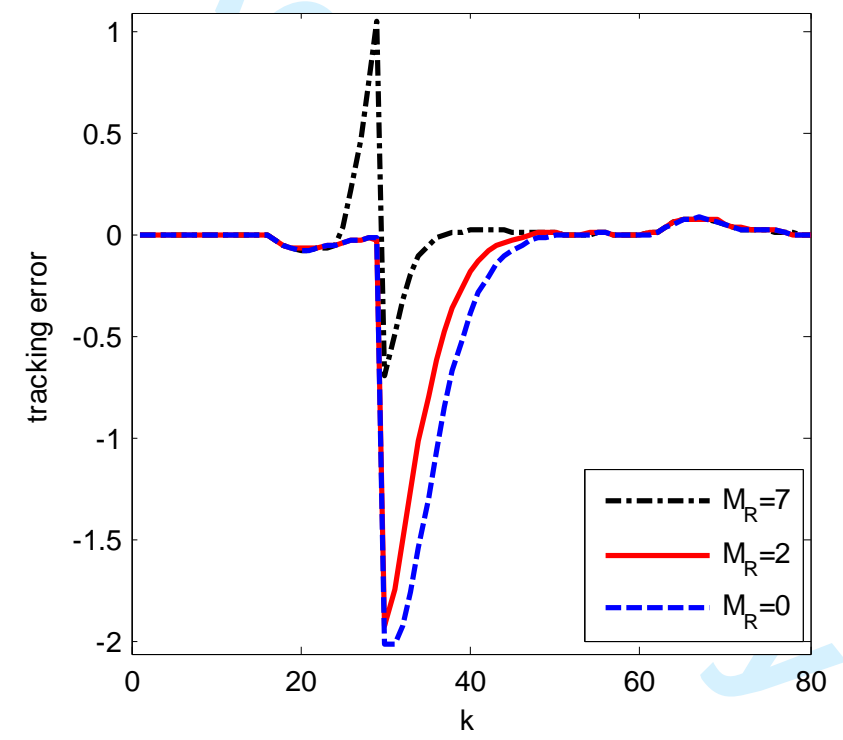

Figure 2. The tracking error of uncertain system to step signal.

In addition, from $(10), S_{x}=\left[\begin{array}{c}1.68977 \\ 1.00000 \\ -5.55781 \\ 0.52632\end{array}\right]$ and $S_{u}=0.05858$ can be obtained. Therefore, the steady-state values of the introduced auxiliary variable $\bar{x}(k)$ and $\bar{u}(k)$ are given by 


$$
\bar{u}(\infty)=\lim _{k \rightarrow \infty} u(k) \approx 0.11716, \bar{x}(\infty)=\lim _{k \rightarrow \infty} x(k) \approx\left[\begin{array}{c}
3.37954 \\
2.00000 \\
-11.11562 \\
1.05264
\end{array}\right]
$$

The simulations shows that the steady-state values of $\bar{x}(k)$ and $\bar{u}(k)$ do tend toward the steady-state values of the nominal system of system (1). And the steady-state values of system (1) are close to the steady-state values of the nominal system over time. Here, as an example, Figure 3 shows the curve of the control input changing in time.

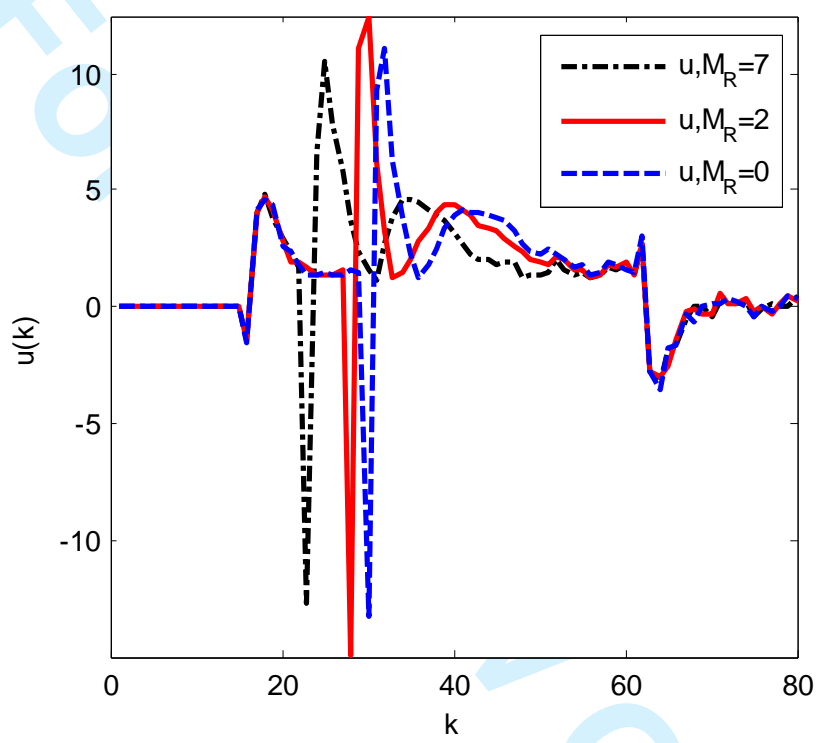

Figure 3. The control input of uncertain system to step signal.

Furthermore, according to Figure 1, it is seen that the closed-loop system has desirable steady-state response characteristics. The output response curve (i.e., Figure 1) will be further analysized by using the dynamic characteristics in the following.

The rise time: $k_{r}=7, k_{r}=9, k_{r}=10$;

the delay time: $k_{d}=29, k_{d}=35, k_{d}=37$;

the settling time: $k_{s}=34, k_{s}=41, k_{s}=44$.

Based on the above, the preview actions can make the closed-loop system has better dynamic characteristics.

(2) The reference signal is the ramp signal 


$$
r(k)=\left\{\begin{array}{cc}
0, & k \leq 10, \\
0.05(k-10), & 10<k<50, \\
2, & k \geq 50 .
\end{array}\right.
$$

For the reference signal (50), the simulation will be completed for the following three situations, that is, (1) $M_{R}=7$, (2) $M_{R}=2$, (3) $M_{R}=0$. Figure 4 indicates the output of system (1) and the desired tracking signal. Figure 5 shows the tracking error and Figure 6 plots the control input, respectively. It can be seen from Figure 4 - Figure 6 that the speed of the output response tracking the reference signal is faster and the adjustment time can be shortened by increasing the preview length of the reference signal.

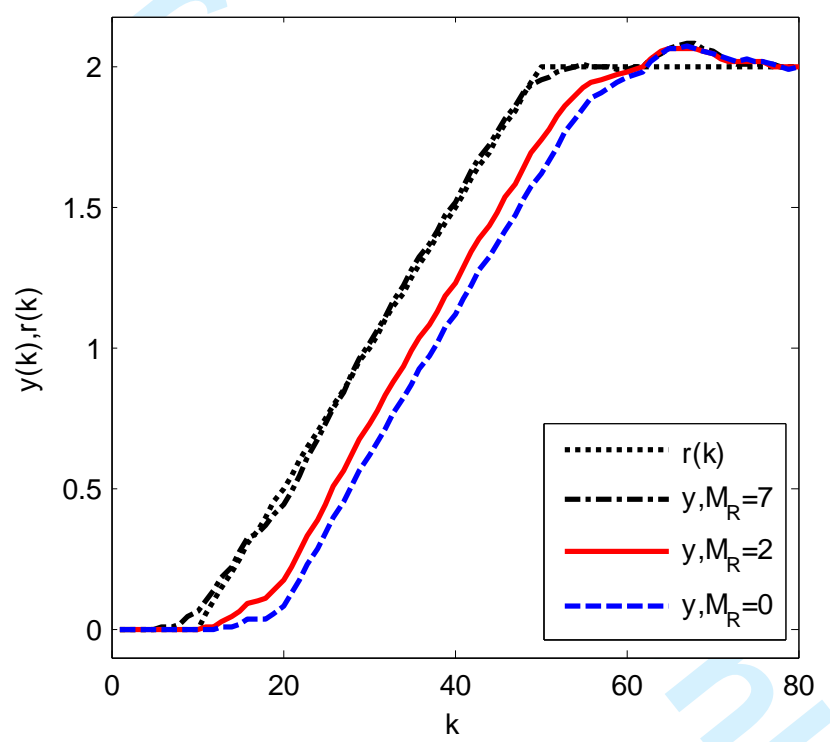

Figure 4. The output response of uncertain system to ramp signal. 


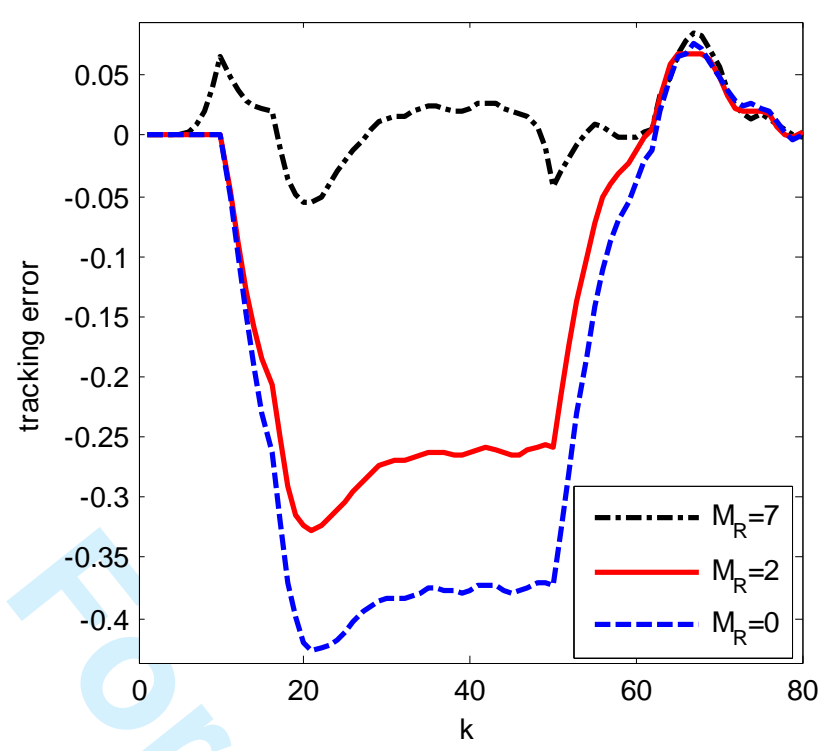

Figure 5. The tracking error of uncertain system to ramp signal.

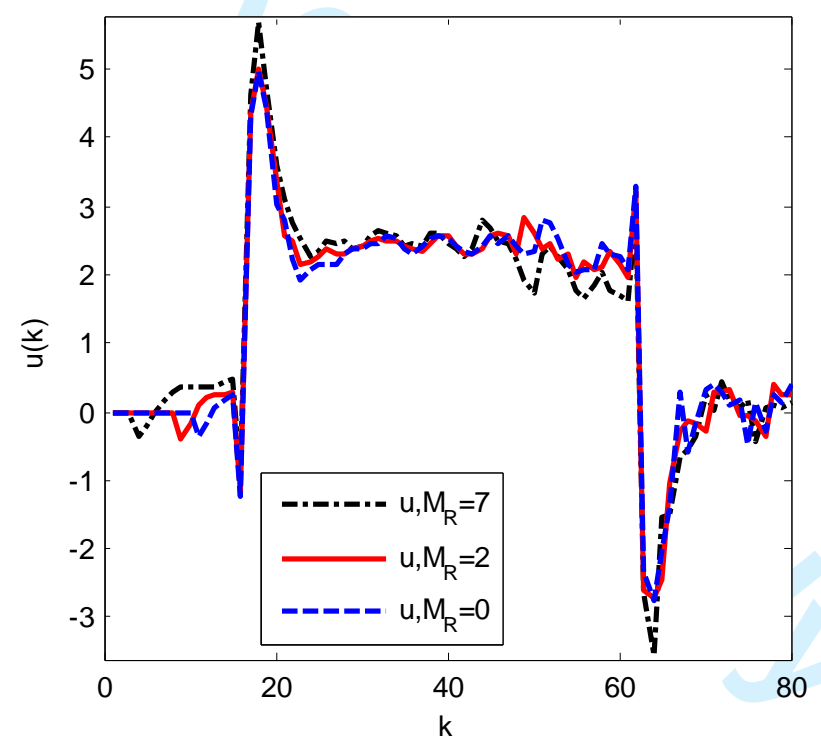

Figure 6. The control input of uncertain system to ramp signal.

It follows from the simulations of periodic interference signals that the design control system still has perfect tracking performance and possesses strong disturbance rejection. And these figures for the results will no longer be presented here due to considering the length of this paper.

To design the observer, let 


$$
L_{1}=\left[\begin{array}{c}
-64.73443 \\
-0.99840 \\
-51.58871 \\
-15.11286
\end{array}\right]
$$

and we obtain

$$
A+L_{1} C A=\left[\begin{array}{cccc}
1.40000 & -57.76099 & 0.20000 & -12.39954 \\
0 & 0.00144 & 0 & 0.00030 \\
-0.24000 & -46.18983 & 0.97000 & -9.78185 \\
0.24000 & -13.84158 & 0.02000 & -1.97144
\end{array}\right] .
$$

As one can easily confirm, $A+L_{1} C A$ is stable. Therefore, the state of system (45) is reconstructed. When the preview length is identical, namely, $M_{R}=7$, Figure 7 indicates the output curves of system (1) without the observer, and with the observer (47).

$$
\text { Let the initial conditions be and } \hat{\tilde{x}}(0)=\left[\begin{array}{c}
0.12 \\
-0.1 \\
0.1 \\
0
\end{array}\right] \text {. From Figure } 7, \text { it can be seen that }
$$

by choosing proper $L_{1}, L_{2}$ and $L_{3}$, the oscillation triggered by the deviation of initial value decays quickly in the process of restructuring the system state with the observer (47). The observer can estimate the state variable quickly and accurately and restrain the disturbance effectively. The effect of control with the observer (47) is ideal.

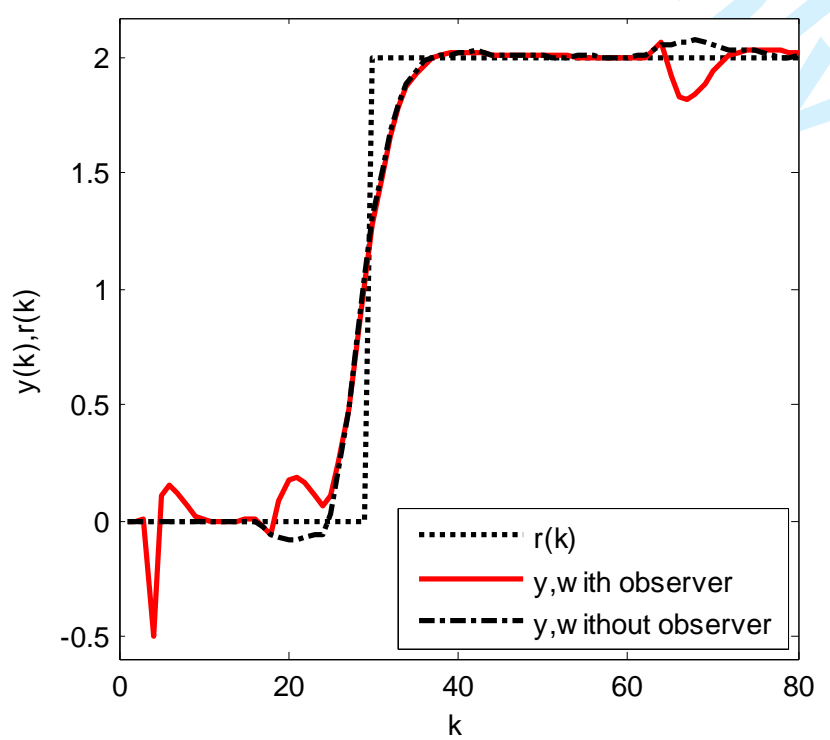


Figure 7: Output response with and without the observer

\section{Conclusion}

By using two new variables related to the system state and input, the method for constructing an augmented error system adopted in this paper overcomes the difficulty faced in previous works [5]. In this manner, the augmented error is simple and easy to handle. And the results of [17-19] can be generalized into a class of systems with general uncertainties. The method used to study the robust preview control by using LMI technique in this paper can be extended completely to other time-invariant or time-varying systems for preview control. The numerical simulation example also illustrated the advantage of the controller with preview action. In our future work, based on an auxiliary approach and LMI techniques, attempts will be made to the output-feedback preview control problem for uncertain systems.

\section{References}

1. Sheridan, T. B., "Three models of preview control," IEEE Trans. Hum. Factors Electron., Vol. 7, No. 2, pp. 91-102 (1966). DOI: 10.1109/THFE.1966.232329

2. Katayama, T., T. Ohki, T. Inoue, and T. Kato, "Design of an optimal controller for a discrete-time system subject to previewable demand," Int. J. Control, Vol. 41, No. 3, pp. 677-699 (1985). DOI: 10.1080/0020718508961156

3. Tomizuka, M., “Optimal continuous finite preview problem," IEEE Trans. Autom. Control, Vol. 21, No. 3, pp. 362-365 (1975). DOI: 10.1109/TAC.1975.1100962

4. Liao, F., Z. Ren, M. Tomizuka, and J. Wu, "Preview control for impulse- free continuous-time descriptor systems," Int. J. Control, Vol. 88, No. 6, pp. 1142-1149 (2015). DOI: 10.1080/00207179.2014.996769

5. Xu, Y. and F. Liao, "Preview control for a class of time-varying discrete systems with input time-delay," Control Decis., Vol. 28, No. 3, pp. 466-470 (2013). DOI: 10.13195/j.cd.2013.03.148.xuyj.023 
6. Yoshimura, T., "Discrete-time adaptive sliding mode controller for vehicle steering systems with preview," J. Vib. Control, Vol. 19, No. 10, pp. 1587-1600 (2013). DOI: 10.1177/1077546312447835

7. Gershon, E. and U. Shaked, " $H_{\infty}$ preview tracking control of retarded statemultiplicative stochastic systems," Int. J. Robust Nonlinear Control, Vol. 24, No. 15, pp. 2119-2135 (2014). DOI: 10.1002/rnc.2979

8. Ozdemir, A. A., P. Seiler, and G. J. Balas, "Design tradeoffs of wind turbine preview control," IEEE Trans. Control Syst. Technol., Vol. 21, No. 4, pp. 1143-1154 (2013). DOI: 10.1109/TCST.2013.2261069

9. Gohrle, C., A. Schindler, A. Wagner, and O. Sawodny, "Design and vehicle implementation of preview active suspension controllers," IEEE Trans. control Syst. Technol., Vol. 22, No. 3, pp. 1135-1142 (2014). DOI: 10.1109/TCST.2013.2272342

10. Shimmyo, S., T. Sato, and K. Ohnishi, "Biped walking pattern generation by using preview control based on three-mass model," IEEE Trans. Ind. Electron., Vol. 60, No. 11, pp. 5137-5147 (2013). DOI: 10.1109/TIE.2012.2221111

11. Kojima, A., " $H_{\infty}$ controller design for preview and delayed systems," IEEE Trans. Autom. Control, Vol. 60, No. 2, pp. 404-419 (2015). DOI: 10.1109/TAC.2014.2354911

12. Moelja, A. A. and G. Meinsma, " $H_{2}$ control of preview systems," Automatica, Vol. 42, No. 6, pp. 945-952 (2006). DOI: 10.1016/j.automatica.2006.01.023

13. Akbari, A. and B. Lohmann, "Output feedback $H_{\circ} / \mathrm{GH}_{2}$ preview control of active vehicle suspension: a comparison study of LQG preview," Vehicle Syst. Dyn., Vol. 48, No. 12, pp. 1475-1494 (2010). DOI: 10.1080/00423110903509327

14. Moran Cardenas, A. J. G. Rázuri, I. Bonet, R. Rahmani, and D. Sundgren, "Design of high accuracy tracking systems with $H_{\infty}$ preview control," Polibits, Vol. 50, pp. 21-28 (2014).

15. Shaked, U. and C. E. de Souza, "Continuous-time tracking problems in an $H_{\infty}$ setting: a game theory approach," IEEE Trans. Autom. Control, Vol. 40, No. 5, pp. 841-852 (1995). DOI: 10.1109/9.384218 
16. Cohen, A. and U. Shaked, "Linear discrete-time $H_{\infty}$-optimal tracking with preview," IEEE Trans. Autom. Control, Vol. 42, No. 2, pp. 270-276 (1997). DOI: $10.1109 / 9.554409$

17. Cohen, A. and U. Shaked, "Robust discrete-time $H_{\infty}$-optimal tracking with preview," Int. J. Robust Nonlinear Control, Vol. 8, No. 1, pp. 29-37 (1998). DOI: 10.1002/(SICI)1099-1239(199801)8:1<29::AID-RNC276>3.0.CO;2-O

18. Takaba, K., "Robust servomechanism with preview action for polytopic uncertain systems," Int. J. Robust Nonlinear Control, Vol. 10, No. 1, pp. 101-111 (2000). DOI: 10.1002/(SICI)1099-1239(200002)10:2<101::AID-RNC465>3.0.CO;2-9

19. Oya, H., K. Hagino, and M. Matsuka, "Observer-based robust tracking control with preview action for uncertain discrete-time systems," IEICE Trans. Fund. Electron. Comm. Comput. Sci., Vol. E90-A, No. 2, pp. 517-522 (2007). DOI: 10.1093/ietfec/e90-a.2.517

20. Liao, F., J. Wang, and G. Yang, "Reliable robust preview tracking control against actuator faults," Asian J. Control, Vol. 5, No. 1, pp. 124-131 (2003). DOI: 10.1111/j.1934-6093.2003.tb00103.x

21. Tsuchiya, T. and T. Egami, Digital Preview and Predictive Control (translated by Liao Fucheng), Beijing Sci. Technol. Press, Beijing (1994).

22. Morais, C. F., M. F. Braga, R. C. L. F. Oliveira, and P. L. D. Peres, “ $H_{\infty}$ state feedback control for MJLS with uncertain probabilities," Automatica, Vol. 52, pp. 317-321 (2015). DOI: 10.1016/j.automatica.2013.01.044

23. $\mathrm{Hu}, \mathrm{H}$. , B. Jiang, and H. Yang, "Robust $H_{\infty}$ reliable control for uncertain switched systems with circular disk pole constraints," J. Frankl. Inst., Vol. 350, No. 4, pp. 803-817 (2013). DOI: 10.1016/jfranklin.2012.12.027

24. Liao, F., T. Katayama, and L. Zheng, "Robust exponential control of a class of systems with uncertainties," J. Uni. Sci. Tech. Beijing, Vol. 9, No. 2, pp. 149-155 (2002).

25. Zhang, Z., Z. Zhang, H. Zhang, P. Shi, and H. Karimi, "Finite-time H filtering for T-S fuzzy discrete-time systems with time-varying delays and norm-bounded uncertainties," IEEE Trans. Fuzzy Sys., Vol. 23, No. 6, pp. 2427-2434 (2015). 
26. Fujisaki, Y. and T. Narazaki, "Optimal preview control based on quadratic performance index," in Proc. 36th IEEE Conf. Decis. Control, San Diego, California USA, pp. 3830-3835 (1997). DOI:10.1109/CDC.1997.652458

27. Egami, T. and T. Tsuchiya, "Disturbance suppression control with preview action of linear DC brushless motor," IEEE Trans. Ind. Electron., Vol. 42, No. 5, pp. 494-500 (1995). DOI: 10.1109/41.464612

28. Kobayashi, E., A. Naganawa, K. Aida, and G. Obinata, "Design of optimal generalized predictive control system," Electr. Eng. Jap., Vol. 134, No. 2, pp. 44-52 (2001). DOI: 10.1541/ieejias.119.1252

29. Liao, F., K. Takaba, T. Katayama, and J. Katsuura, "Design of an optimal servomechanism for discrete-time systems in a multirate setting," Dyn. Contin. Discrete Impuls. Syst. Ser. B: Appl. Algorithms, Vol. 10, No. 5, pp. 727-744 (2003).

30. Chang, X. and G. Yang, "New results on output feedback $H_{\infty}$ control for linear discrete-time systems," IEEE Trans. Autom. Control, Vol. 59, No. 5, pp. 1355-1359 (2014). DOI: 10.1109/TAC.2013.2289706

31. Li, J., J. Wu, and L. Fan, "Design of $H_{\infty}$ reduced-order controller for discrete system," Electr. Mach. Control, Vol. 15, No. 6, pp. 78-83 (2011). DOI: 10.15938/j.emc.2011.06.006

32. Lee, K. H., J. H. Lee, and W. H. Kwon, "Sufficient LMI conditions for $H_{\infty}$ output feedback stabilization of linear discrete-time systems," IEEE Trans. Autom. Control, Vol. 51, No. 4, pp. 675-680 (2006). DOI: 10.1109/TAC.2006.872766

33. Guo, X. and G. Yang, "Insensitive output feedback $H_{\infty}$ control of delta operator systems with insensitivity to sampling time jitter," Int. J. Robust Nonlinear Control, Vol. 24, No. 4, pp. 725-743 (2014). DOI:10.1002/rnc.2915

34. Chen, Y., S. Fei, and Y. Li, "Stabilization of neutral time-delay systems with actuator saturation via auxiliary time-delay feedback," Automatica, Vol. 52, pp. 242-247 (2015). DOI: 10.1016/j.automatica.2014.11.015

35. He, Y., M. Wu, and J. She, "Improved bounded-real-lemma representation and $H_{\infty}$ control of systems with polytopic uncertainties," IEEE Trans. Circuits Syst. 
II, Exp. Briefs, Vol. 52, No. 7, pp. 380-383 (2005). DOI: 10.1109/TCSII.2005.850418

36. Zheng, D., Linear System Theory, Second Edition, Tsinghua Univ. Press, Beijing (2012).

37. Gong, D., Discrete Control System Theory Introduction, China Railway Press, Beijing (2004).

38. Liao, F. and P. Chen, "Optimal preview control based on state observers for linear discrete- time systems," J. Univ. Sci. Technol. Beijing, Vol. 36, No. 3, pp: 390-398 (2014). DOI: 10.13374/j.issn1001--053x.2014.03.018

39. Li, L. and F. Liao, "Design of a preveiw controller for discrete-time systems based on LMI,” Math. Probl. Eng., Vol. 2015, pp. 1-12 (2015). DOI: 10.1155/2015/179126 


\section{Response to Editor and Reviewers}

Dear Dr. Li-Chen Fu and Reviewers:

Thanks very much for your careful review and constructive suggestions on the manuscript " $H_{\infty}$ Preview Control of a Class of Uncertain Discrete-Time Systems". We have revised the manuscript carefully according to reviewers' comments. The detailed revisions are listed below based on reviewers' response point by point. We really hope that the revised version could satisfy all requirements of the $3 \mathrm{rd}$ reviewer.

Best wishes to you!

Yours sincerely,

Fucheng Liao 
1. I would suggest that the class of systems to which the approach is applicable is stated in the abstract.

Ans. Thanks for pointing this out. In response to the 3rd reviewer's suggestion, the class of systems to which the approach is applicable has been stated in the revised manuscript.

2. The answers to some comments will be addressed in future works of the authors: comment 1 of reviewer 2 , comment 4 of reviewer 3 . I find the answers not convincing.

Ans. Your suggestion is greatly appreciated. An auxiliary approach is used to derive the augmented error system of uncertain discrete-time system to transform the tracking control problem into a robust $H_{\infty}$ control problem. In designing robust preview controller, for the augmented error system, the state-feedback is introduced under the assumption that the state variables of the original system are available. Then the gain matrix can be obtained by solving the feasible solution problem of linear matrix inequality. Finally by putting the controller obtained into the original system, we can get the preview controller (see Theorem 4). And the preview controller for system (1) is given by

$$
u(k)=K_{e} e(k)+K_{x} \tilde{x}(k)+\sum_{i=0}^{M_{R}} K_{R}(i) r(k+i)+K_{v}\left(\sum_{s=0}^{k-1} e(s)+v(0)\right)+S_{u} r(k) .
$$

Subsequently, if the state variables of the original system cannot be measured as feedback state variables, equivalently $\tilde{x}(k)$ cannot be used in equation (1) and then the states of system (25) cannot be available. Based on these, a state observer for preview control systems is introduced to reconstruct $\tilde{x}(k)$. The state observer (i.e., (47)) is a full-order dimensional observer with respect to the original system whereas it is a reduced-order observer with respect to the augmented error system. Then based on the reduced-order observer design method in linear system theory, the state observer is obtained. $\hat{\tilde{x}}(k)$ can be obtained by (47) (i.e., $\hat{\tilde{x}}(k)=W(k)-L Z(k))$ and the preview controller is given by 


$$
u(k)=K_{e} e(k)+K_{x} \hat{\tilde{x}}(k)+\sum_{i=0}^{M_{R}} K_{R}(i) r(k+i)+K_{v}\left(\sum_{s=0}^{k-1} e(s)+v(0)\right)+S_{u} r(k) .
$$

It should be pointed out that the observer is designed without using $H_{\infty}$ control. More detail on this issue has been presented in section 5. And the problem of output feedback preview controller isn't considered in this manuscript. However, the problems of the output feedback $H_{\infty}$ preview control have been studied. Part of this work has been published in the following literature:

Li Li and Fucheng Liao. Parameter-dependent preview control with robust tracking performance. IET Control Theory and Application, 1-27.

DOI: 10.1049/iet-cta.2016.0361, Print ISSN 1751-8644, Available online: 26 September 2016.

For comment 4 of reviewer 3: In response, the convex feasibility problem has been converted into the convex optimal control problem. In other words, $\gamma$ is considered as a decision variable which should be minimized rather than a given value in $H_{\infty}$ design problems. And the numerical example (section 6) has been revised accordingly and the simulation results are better than before.

3. On comment 5 of reviewer 3: isn't it possible to perform the design for system without uncertainty and do the analysis (comparison) for uncertain system?

Ans. Thanks for your comments. For the linear system without uncertainty, the usual difference method is used to construct an augmented error system in classical preview control including previewed information. By applying the difference operator to the equation of the states, error vectors and predictable reference signals, the tracking control problem of the original systems is turned into generalized-expanded error systems. Specific derivation has presented in reference [2, 5, 6, 21].

However, for discrete-time systems with time-varying uncertainties, the difference operator $\Delta$ (i.e., $\Delta x(k)=x(k+1)-x(k)$ or $\Delta x(k+1)=x(k+1)-x(k))$ cannot be applied to both sides of uncertain system (1) directly. In other words, the difference operator cannot be employed for the uncertainties which are often 
time-varying and unknown, or may be nonlinear. Therefore, uncertainties become the main roadblock in applying the method of augmented error systems in classical preview control. Our proposed method is derived to construct an augmented error system instead of usual taking the difference of the error signal and the system equation. The new approach avoids applying the difference operator to the time-varying matrix and can simplify the augmented error system. And this method not only is applicable to the systems without uncertainty, but also can be used for uncertain system with general form. 